other case of spontaneous CSF leak, 4 leakage sites were suspected; patient underwent 4 epidural blood and fibrin glue patches at various levels between T1 and L4, with none of which was successful in controlling the symptoms. Again, a PICC line inserted at L3/4 level was navigated to the craniocervical junction followed by fibrin glue injection in the epidural space from the craniocervical junction down to the upper lumbar level. The procedure was successful in achieving symptoms resolution. No post-procedure complications occurred in both patients.

Conclusions Epidural Fibrin glue injection using peripherally inserted central catheter (PICC) lines is a safe and effective approach for treatment of refractory CSF leaks with multiple or unconfirmed sites of leakage.

Disclosures R. Abdalla: None. D. Cantrell: None. M. Hurley: None. S. Ansari: None. A. Shaibani: None.

\section{E-143 EMBOLIZATION OF SPINAL DURAL ARTERIOVENOUS FISTULA: NBCA IS SUPERIOR TO ONYX EMBOLIZATION}

J Baranoski*, J Catapano, T Cole, N Majmundar, B Hendricks, D Wilkinson, A See, D Cavalcanti, B Flores, A Ducruet, F Albuquerque. Neurosurgery, The Barrow Neuroloigcal Institute, Phoenix, $A Z$

\subsection{6/neurintsurg-2020-SNIS.175}

Introduction/Purpose Historically, spinal dural arteriovenous fistulas (SDAVFs) have been treated with intradural ligation of the draining vein. While endovascular embolization can achieve durable occlusion in a subset of these patients, recent studies have demonstrated a superiority of microsurgical ligation. However, given that spinal angiography is essential in the workup of suspected SDAVFs, we and others have demonstrated the utility of an 'endovascular-first' approach to SDAVFs. One limiting factor to the success of endovascular therapies has been the reliability of casting and occluding the draining vein using Onyx. In contrast, we have observed that n-butyl-2-cyanoacrylate (nBCA) glue can readily penetrate and cast the fistula and vein. We therefore sought to compare the efficacy of nBCA and Onyx embolization in the treatment of SDAVFs.

Material and Methods We performed a retrospective analysis of our endovascular database from 4/2007 to 4/2019 to identify patients treated for SDAVFs. We extracted demographic, clinical, treatment, and radiographic information data. We considered a 'durable cure' of the SDAVF to include complete obliteration of the fistula following endovascular treatment, demonstrated clinical improvement, and follow-up radiographic studies demonstrating improvement of spinal cord edema and flow voids on MRI/MRA and/or persistent obliteration on follow-up angiograms. We compared our results using Onyx and nBCA embolysate.

Results We performed 40 endovascular treatments for SDAVFs in 38 patients. The embolysates utilized were Onyx without any other embolysate (22), NBCA without any other liquid embolysate (16), and a combination of nBCA and Onyx (2). For the 22 treatments in which Onyx was the only embolysate used, endovascular treatment resulted in a durable cure in 11 cases (50\%) (mean follow-up 20.8 months). For 10 of these 22 (45.5\%), a complete obliteration of the fistula with casting of the vein was not achieved and these patients were referred for microsurgical ligation. For 1 of these 22 treatments, a complete obliteration of the fistula was noted on immediate post-procedure angiography. However, 4.2 months after treatment, he was found to have a recurrence. For the 16 treatments in which nBCA was the only liquid embolysate used, endovascular treatment resulted in a complete obliteration of the fistula following treatment in all $16(100 \%)$ patients. None of these patients underwent subsequent microsurgical treatment. Follow-up data were available for 13 of these patients $(81.3 \%)$. Of these 13 , all (100\%) achieved a durable cure (mean follow-up 19.6 months) without clinical or radiographic recurrence. A durable cure was achieved more frequently in patients treated with $\mathrm{nBCA}(100 \%)$ relative to those treated with Onyx only $(50 \%)(\mathrm{p}<0.01)$. We evaluated the fluoroscopy time used in cases where obliteration of the fistula was achieved using a single radicular artery and resulted in a durable cure. When Onyx was used, the mean fluoroscopy time was 50.8. In contrast, the mean fluoroscopy time was significantly shorter $(\mathrm{P}<0.02)$ when nBCA was used for these cases (mean 31.8 minutes).

Conclusion NBCA embolization of SDAVFs is superior to Onyx embolization. NBCA embolization is safe and effective for a subset of SDAVFs with results in this population comparable to microsurgical ligation.

Disclosures J. Baranoski: None. J. Catapano: None. T. Cole: None. N. Majmundar: None. B. Hendricks: None. D. Wilkinson: None. A. See: None. D. Cavalcanti: None. B. Flores: None. A. Ducruet: None. F. Albuquerque: None.

\section{E-144 MINIMALLY INVASIVE SCOLIOSIS SURGERY WITH TRANS KAMBIN POSTERIOR OBLIQUE LATERAL LUMBAR INTERBODY FUSION: SINGLE SURGEON FEASIBILITY STUDY}

H Abbasi*. Neurosurgery, Inspired Spine, Alexandria, MN

\subsection{6/neurintsurg-2020-SNIS.176}

Background Degenerative deformities of the spine have traditionally been treated with extensive open surgeries. However, these open procedures are associated with a high degree of surgical morbidity. In this study, we explore whether clinical improvement in patients with spinal deformities can be achieved using a new minimally invasive surgery (MIS) called oblique lateral lumbar interbody fusion (OLLIF). OLLIF is a MIS single surgeon procedure in which the disc is approached through Kambin's triangle. OLLIF can achieve correction of spinal deformities through careful cage placement.

Purpose The purpose of this study is to establish the safety and efficacy of using OLLIF to correct spinal deformities and to collect early outcome data. Collected data includes perioperative outcomes, patient reported outcomes, and radiographic outcomes.

Study Design/Setting This study is a retrospective review of 37 OLLIF surgeries in 36 patients with symptomatic degenerative spinal deformity. Collected perioperative data included surgery time, blood loss, and hospital stay. Follow-up was conducted at least 150 days post surgery. We recorded complications and patient reported outcomes such as Oswestry Disability Index (ODI) and pain scale. Imaging was conducted pre- and postsurgery. Fusion rates and changes in Cobb angle were also measured.

Results A total of 37 surgeries that treated 100 vertebral levels were performed. For two and three level procedures, respectively, the mean blood loss was 83 and $178 \mathrm{ml}$, the average surgery time was 74 and 158 minutes and the 